\title{
Order with successors is not interpretable in RCF
}

\author{
by
}

\author{
S. Świerczkowski (Muscat)
}

\begin{abstract}
Using the monotonicity theorem of L. van den Dries for RCF-definable real functions, and a further result of that author about RCF-definable equivalence relations on $\mathbb{R}$, we show that the theory of order with successors is not interpretable in the theory RCF. This confirms a conjecture by J. Mycielski, P. Pudlák and A. Stern.
\end{abstract}

1. Let RCF be the theory of real closed fields. We may view RCF as the first order theory of the structure $\langle\mathbb{R} ;+, \cdot, \leq, 0,1\rangle$, where $\mathbb{R}$ is the set of real numbers and,$+ ;, \leq, 0,1$ have the usual meaning. It is conjectured in $[6],\left(\mathrm{P}_{23}\right)$, that the order theory of $\omega=\{0,1,2, \ldots\}$, i.e., $\operatorname{Th}(\langle\omega ; \leq\rangle)$, cannot be interpreted in RCF:

$$
|\mathrm{Th}(\langle\omega ; \leq\rangle)| \not \leq|\mathrm{RCF}| .
$$

Here $|T|$ denotes the chapter of mathematics containing a given theory $T$ (i.e., the class of all theories which both interpret and are interpretable in $T)$. $\left|T_{1}\right| \leq\left|T_{2}\right|$ means that $T_{1}$ is interpretable in $T_{2}$.

Our aim in this note is to prove (1.1). Familiarity with the definition of interpretability, as proposed by Jan Mycielski in [5], will be assumed. For further information, the reader is referred to the survey [6].

It is known that the order theories of $\omega$ and $\mathbb{Q}$ are not interpretable in each other: Both non-interpretability results

$$
|\operatorname{Th}(\langle\omega ; \leq\rangle)| \not \leq|\operatorname{Th}(\langle\mathbb{Q} ; \leq\rangle)| \text { and }|\operatorname{Th}(\langle\mathbb{Q} ; \leq\rangle)| \not \leq|\operatorname{Th}(\langle\omega ; \leq\rangle)|
$$

were obtained by J. Krajičck [4]. (The second part of (1.2) was proved independently by A. Stern; a proof which combines the methods of both authors is given in [8].) Obviously

$$
|\operatorname{Th}(\langle\mathbb{Q} ; \leq\rangle)| \leq|\mathrm{RCF}|,
$$

so, using the second part of (1.2), we conclude that RCF is not interpretable in $\operatorname{Th}(\langle\omega ; \leq\rangle)$. Hence, by $(1.1)$, the chapters $|\mathrm{RCF}|$ and $|\operatorname{Th}(\langle\omega ; \leq\rangle)|$ are incomparable. The first part of (1.2), easier to establish, clearly also follows from (1.1) and (1.3). 
Since $|\operatorname{Th}(\langle\mathbb{Z} ; \leq\rangle)|=|\operatorname{Th}(\langle\omega ; \leq\rangle)|$, we may replace $\omega$ by $\mathbb{Z}$ in the above considerations. Another consequence of (1.1) is the well known fact that $\omega$ is not an RCF-definable subset of $\mathbb{R}$ (stemming from the undecidability of arithmetic).

To state our result quite exactly, let us specify first the axioms of a preordering with successors. We shall call a binary relation $\ll$ a pre-ordering if $\ll$ is reflexive, transitive and there is universal comparability:

$$
\forall x \forall y(x \ll y \vee y \ll x) .
$$

For any such pre-ordering we abbreviate $(x \ll y) \wedge(y \ll x)$ as $x \approx y$. Clearly, $\approx$ is an equivalence relation; we call it the equivalence relation associated with $\ll$. We denote by $\operatorname{Succ}(x, y)$ the formula

$$
x \ll y \wedge x \not \approx y \wedge \forall z(x \ll z \ll y \rightarrow z \approx x \vee z \approx y),
$$

reading it: " $y$ is an immediate successor of $x$ ". We say that $\ll$ is a pre-ordering with successors if every $x$ has an immediate successor, i.e., if $\forall x \exists y \operatorname{Succ}(x, y)$.

It is clear that (1.1) will result if we prove:

THEOREM 1.1. Any sentence which proves the existence of a pre-ordering with successors is not interpretable in $R C F$.

We take this opportunity to mention another recent result about RCF, and to pose a problem. A theory $T$ is called connected if $T$ interprets in the union $T_{1} \cup T_{2}$ of two theories with disjoint languages only if $T$ interprets in $T_{1}$ or in $T_{2}$. The problem if RCF is connected was raised in [6], $\left(\mathrm{P}_{2}\right)$. A positive answer was found recently by A. Stern and the author [9].

A theory $T$ is called compact if there is a finitely axiomatizable theory $T^{\prime}$ such that $|T|=\left|T^{\prime}\right|$, i.e., $T$ and $T^{\prime}$ interpret each other.

Problem. Is RCF compact?

2. A subset $S$ of $\mathbb{R}^{d}, d \geq 1$, will be called definable if $S$ is definable in the language $\{+, \cdot, \leq, 0,1\}$ of $\mathrm{RCF}$, i.e., if there is a formula $\phi$ in that language and there are some $a_{1}, \ldots, a_{k} \in \mathbb{R}$ such that, for all $x_{1}, \ldots, x_{d} \in \mathbb{R}$,

$$
\left(x_{1}, \ldots, x_{d}\right) \in S \leftrightarrow \phi\left(x_{1}, \ldots, x_{d}, a_{1}, \ldots, a_{k}\right) .
$$

Functions and relations on Cartesian powers of $\mathbb{R}$ will be called definable if they have definable graphs. We shall show that for every $d \geq 1$, there does not exist on $\mathbb{R}^{d}$ a definable relation of pre-ordering with successors. Clearly, this will be enough to establish Theorem 1.1. The following known facts will be needed in the proof.

THEOREM $2.1([1])$. Given a definable equivalence relation $\approx$ on $\mathbb{R}^{d}(d \geq$ $1)$, there is a definable function $f: \mathbb{R}^{d} \rightarrow \mathbb{R}^{d}$ which selects one representative 
from each $(\approx)$-equivalence class, i.e., such that for any d-tuples $\bar{x}, \bar{y} \in \mathbb{R}^{d}$,

$$
\bar{x} \approx \bar{y} \leftrightarrow f(\bar{x})=f(\bar{y}) \text { and } \bar{x} \in f(\bar{x}) .
$$

The next theorem is proved, in the general setting of $O$-minimal structures, in [7]:

TheOREM 2.2 (Monotonicity Theorem in [2], [3]). For every definable function $f: \mathbb{R} \rightarrow \mathbb{R}$ there is a partition of $\mathbb{R}$ into finitely many points and open intervals such that on each of the intervals $f$ is either constant or strictly monotone and continuous.

So, for a definable $f: \mathbb{R} \rightarrow \mathbb{R}$ there are only finitely many $y \in \mathbb{R}$ such that the pre-image $f^{-1}(y)$ is infinite. Combining this observation with the case $d=1$ of Theorem 2.1, we get:

LEMMA 2.3. Every definable equivalence relation on $\mathbb{R}$ has only finitely many infinite equivalence classes.

3. We need two more lemmas:

LEMMA 3.1. Let $L_{0} \subset L_{1} \subset L_{2} \subset \ldots$ be a strictly increasing sequence of subsets of $\mathbb{R}$ such that each boundary $\partial L_{i}$ is a finite set. Suppose further that the number of elements \# $\left(\partial L_{i}\right)$ of any of these boundaries does not exceed a fixed constant $K<\infty$. Then the difference $L_{i+1} \backslash L_{i}$ is infinite for infinitely many $i$.

Proof. Suppose there is an $i$ such that $L_{j+1} \backslash L_{j}$ is finite for all $j \geq i$. Let $j>i$ and consider any $x \in L_{j} \backslash L_{i}$. If $x$ is in the interior of $L_{j}$, then, as $x \notin L_{i}$, we must have $x \in \partial L_{i}$, for otherwise a neighbourhood of $x$ would be disjoint from $L_{i}$, and $L_{j} \backslash L_{i}$ would be infinite. So

$$
L_{j} \backslash L_{i} \subseteq \partial L_{i} \cup \partial L_{j}
$$

Since there are at least $j-i$ elements in $L_{j} \backslash L_{i}$, we get

$$
j-i \leq \#\left(\partial L_{i}\right)+\#\left(\partial L_{j}\right) \leq 2 K
$$

which is obviously impossible for all $j \geq i$.

LEMMA 3.2. There does not exist a definable pre-ordering of $\mathbb{R}$ for which there is an infinite sequence $x_{0}, x_{1}, x_{2}, \ldots$ such that every $x_{i+1}$ is an immediate successor of $x_{i}$.

Proof. Suppose that $\ll$ is a definable pre-ordering of $\mathbb{R}$ and $\left(x_{i}\right)_{i<\omega}$ is a sequence such that $\operatorname{Succ}\left(x_{i}, x_{i+1}\right)$ holds for each $i$. By the TarskiSeidenberg quantifier elimination theorem for RCF, the formula $x \ll y$ is RCF-equivalent to a Boolean combination of atomic formulas:

$$
x \ll y \leftrightarrow \bigvee_{j=1}^{r}\left[\left(p_{j}(x, y)=0\right) \wedge \bigwedge_{k=1}^{n}\left(q_{k}^{(j)}(x, y)>0\right)\right],
$$


where $p_{j}(x, y), q_{k}^{(j)}(x, y)$ are real polynomials in two variables. Let us check that if $L_{i}=\left\{x: x \ll x_{i}\right\}, i=0,1,2, \ldots$, then all assumptions of Lemma 3.1 are satisfied. Putting $y=x_{i}$ in the above Boolean combination, we conclude that each $L_{i}$ is a finite union of intervals, so $\partial L_{i}$ is a finite set. Let $a \in \partial L_{i}$. Then, for some $j$, there are numbers $x$, arbitrarily close to $a$, satisfying

$$
\left(p_{j}\left(x, x_{i}\right)=0\right) \wedge \bigwedge_{k=1}^{n}\left(q_{k}^{(j)}(x, y)>0\right) \text {. }
$$

Clearly $p_{j}\left(a, x_{j}\right)=0$. So, if $p_{j}\left(x, x_{i}\right)$ does not vanish identically as a polynomial in $x$, then the number of possible values for $a \in \partial L_{i}$ does not exceed the degree with respect to $x$ of the polynomial $p_{j}(x, y)$. If $p_{j}\left(x, x_{i}\right) \equiv 0$ as a polynomial in $x$, then $a \in \partial L_{i}$ implies that $q_{k}^{(j)}\left(a, x_{i}\right)=0$ for at least one $k$. We conclude that the number of elements of any $\partial L_{i}$ is not greater than the sum of the degrees with respect to $x$ of all the polynomials $p_{j}(x, y)$, $q_{k}^{(j)}(x, y)$, i.e., there is a common finite bound $K$ for the number of elements of each boundary $\partial L_{i}$.

If $\approx$ is the equivalence relation associated with $\ll$ then it is clear that the $(\approx)$-equivalence class $\left[x_{i+1}\right]$ of $x_{i+1}$ equals $L_{i+1} \backslash L_{i}$. Thus each inclusion $L_{i} \subset$ $L_{i+1}$ is proper and we can apply Lemma 3.1, which tells us that infinitely many equivalence classes $\left[x_{i}\right]$ are infinite. But this contradicts Lemma 2.3.

4. Proof of Theorem 1.1. Suppose that $\alpha$ is a sentence which proves the existence of a pre-ordering with successors and $\alpha$ can be $d$-dimensionally interpreted in RCF. Then there exists a definable pre-ordering $\ll$ of $\mathbb{R}^{d}$ with successors. If $d=1$, we get a contradiction with Lemma 3.2, and we are done. Otherwise, consider the equivalence relation on $\mathbb{R}^{d}$ associated with $\ll$ and a definable map $f: \mathbb{R}^{d} \rightarrow \mathbb{R}^{d}$ which selects one representative from each equivalence class, as in Theorem 2.1. We put $E=f\left(\mathbb{R}^{d}\right)$ and denote by $\ll_{E}$ the restriction of $\ll$ to $E$. Clearly $\ll_{E}$ is an ordering of $E$ where each element has a unique immediate successor. Next we choose $e_{0} \in E$, and denote by $\left(e_{i}\right)_{i<\omega}$ the sequence of elements of $E$ such that each $e_{i+1}$ is the immediate successor of $e_{i}$.

By Baire's Category Theorem, there is a straight line in $\mathbb{R}^{d}$ such that all $e_{i}, 0 \leq i<\omega$, have different orthogonal projections on that line. In other words, there is a $w \in \mathbb{R}^{d}$ such that, if we denote for each $x \in \mathbb{R}$ by $H_{x}$ the hyperplane $\{v: v \cdot w=x\}$ in $\mathbb{R}^{d}$, we never get two distinct $e_{i}, e_{j}$ in one such hyperplane. We put now $E^{+}=\left\{e \in E: e_{0} \ll_{E} e\right\}$ and define a pre-ordering $\leq$ of $\mathbb{R}$ by

$$
x \leq y \leftrightarrow\left(H_{x} \cap E^{+}=\emptyset\right) \vee \forall\left(u \in H_{y} \cap E^{+}\right) \exists\left(v \in H_{x} \cap E^{+}\right)\left(v \ll_{E} u\right) .
$$

This gives a definable pre-ordering of $\mathbb{R}$. Denoting by $x_{i}$ the unique $x \in \mathbb{R}$ for which $e_{i} \in H_{x}$, we check that, for every $0 \leq i<\omega, x_{i+1}$ is the immediate 
successor of $x_{i}$. We have again reached a contradiction with Lemma 3.2, and this shows that a $d$-dimensional interpretation of $\alpha$ in $\mathrm{RCF}$ cannot exist.

\section{References}

[1] L. van den Dries, Algebraic theories with definable Skolem functions, J. Symbolic Logic 49 (1984), 625-629.

[2] —, Definable sets in O-minimal structures, lecture notes at the University of Konstanz, spring 1985.

[3] -, Tame Topology and O-minimal Structures, book in preparation.

[4] J. Krajíček, Some theorems on the lattice of local interpretability types, Z. Math. Logik Grundlag. Math. 31 (1985), 449-460.

[5] J. Mycielski, A lattice connected with relative interpretability of theories, J. Symbolic Logic 42 (1977), 297-305.

[6] J. Mycielski, P. Pudlák and A. Stern, A lattice of chapters of mathematics, Mem. Amer. Math. Soc. 426 (1991).

[7] A. Pillay and C. Steinhorn, Definable sets in ordered structures I, Trans. Amer. Math. Soc. 295 (1986), 565-592.

[8] A. Stern, The lattice of local interpretability of theories, Ph.D. Thesis, University of California, Berkeley, March 1984.

[9] A. Stern and S. Świerczkowski, A class of connected theories of order, J. Symbolic Logic, to appear.

DEPARTMENT OF MATHEMATICS AND COMPUTING

P.O. BOX 36 - AL KHOD - PC123

SULTAN QABOOS UNIVERSITY

MUSCAT, SULTANATE OF OMAN

Received 5 March 1993 